\title{
Design of a 2-finger hand exoskeleton for finger stiffness measurements
}

\author{
Angelo Emanuele Fiorilla ${ }^{\mathrm{a}, \mathrm{b} *}$, Nikos G. Tsagarakis ${ }^{\mathrm{b}}$, Francesco Nori ${ }^{\mathrm{b}}$ and Giulio Sandini ${ }^{\mathrm{b}}$ \\ ${ }^{a}$ DIST - University of Genova, Genova, Italy; ${ }^{b}$ Italian Institute of Technology (IIT), Genova, Italy \\ (Received 8 October 2008; final version received 22 March 2009)
}

\begin{abstract}
Recent studies of human arm movements have suggested that the control of stiffness may be important both for maintaining stability and for achieving differences in movement accuracy. Several studies in the robotic field demonstrated that grasp stiffness is useful for modelling and controlling manipulators but, even though it is accredited that having models of the human finger impedance would be very desirable for the control of anthropomorphous robot's hands, relatively few studies have focused on finger and hand stiffness. To allow the measurement of such entities at the finger level, an appropriate device capable of applying fast force transients while at the same time be able to monitor the finger movements is required. The work presented in this paper is a very detailed report about the design of a new hand exoskeleton system that will be used in our future works to investigate the finger stiffness range in different grasping postures and conditions.
\end{abstract}

Keywords: finger stiffness measurements; impedance modelling; dexterous manipulation; physical human-robot interaction; rehabilitation robotics

\section{Introduction}

Previous studies on human arm movements have shown that stiffness - the change in resistive force that counteracts the effect of external loads - is substantially dependent on variability in limb geometry and orientation and on antagonist muscles co-contraction (Gribble et al. 2003). The control of joint impedance provides mechanical stability in the presence of external perturbations and this is the reason why active modulation of limb impedance by the central nervous system (CNS) is considered an essential part of effective motor control (Mussa-Ivaldi et al. 1985; Hogan 1990). At present, relatively little is known about the conditions under which the CNS modulates limb impedance by way of co-contraction or how central commands related to co-contraction are coordinated with those connected to movement production. Previous human studies of mechanical impedance, including single-joint measurements of the ankle (Hunter and Kearney 1983), elbow (Jones and Hunter 1990; Bennett et al. 1992) and multi-articular measurements of the arm (Hogan 1990) and leg (McMahon 1984) have only shown that subjects are able to modulate the coactivation of antagonist muscles around a joint to minimise the perturbing effect of external loads (Kearney and Hunter 1990; Serres and Milner 1991; Latash 1992; Milner and Cloutier 1998; Milner 2002).

At the same time, parallel studies, mechanical analyses and robotic experiments have demonstrated that the appropriate selection of active impedance facilitates the execution of contact tasks (Whitney 1982; Asada and Asari 1988).

Going into the details of manipulation exercises, it has been shown that both the stability and the manipulability of a grasp are affected by the grasp impedance (see Friedman and Flash 2007). Human and dexterous robotic hands have the ability to grasp arbitrary objects and impart different motions and forces to them. They also exhibit compliance resulting from a combination of structural elasticity and control; this compliance helps the fingers to accommodate adjustments in grasping conditions due to unexpected disturbances and/or sudden changes in task requirements. In the specific case of human grasping, while the structural compliance is passive and changes as a function of the orientation of the fingers, an example of active position control is the automatic increase of the grip force to prevent a grasped object from sliding when the object is pulled down by an external force (see Section 2).

Previous studies on modelling human grasps investigated the stiffness of the human hand using different kinds of devices (Figure 1). Some of the experiments were performed using vibrational devices (Fu and Oliver 2005) or platforms actuated by pneumatic (Hajian and Howe 1997) and electrical motors (Kao et al. 1997; Milner and Franklin 1998), while other studies employed commercial exoskeletons (Friedman and Flash 2007).

Some of these works considered the impedance of the different joints of the hand, while other studies measured

\footnotetext{
*Corresponding author. Email: emanuele.fiorilla@iit.it
} 


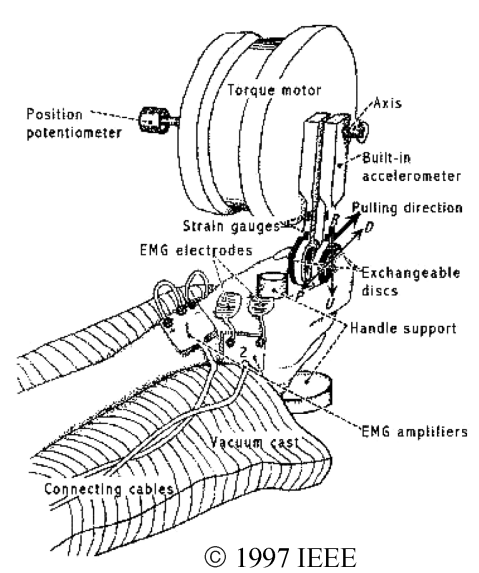

(a) Kao et al.(1997)

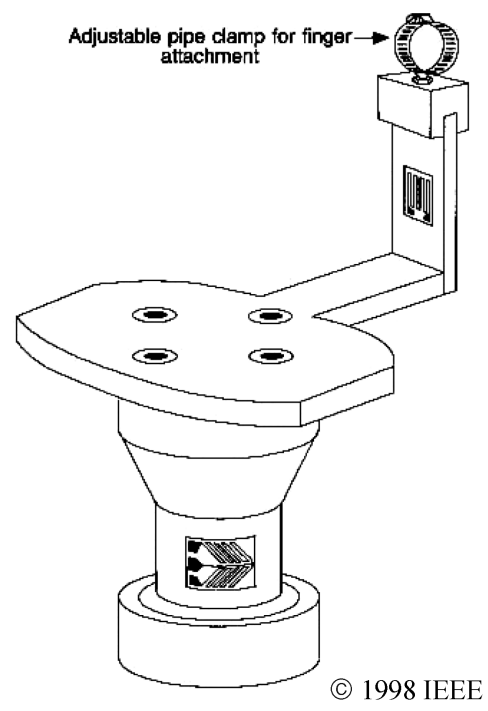

(b) Milner and Franklin (1998)

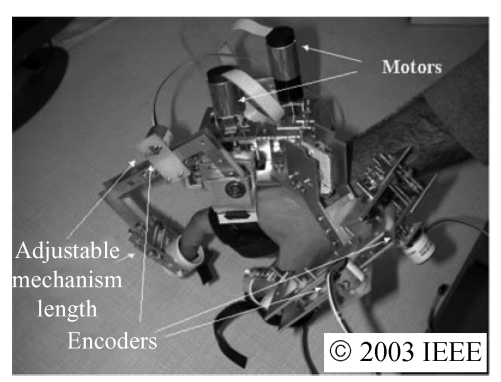

(c) Stergiopoulos et al. (2003)

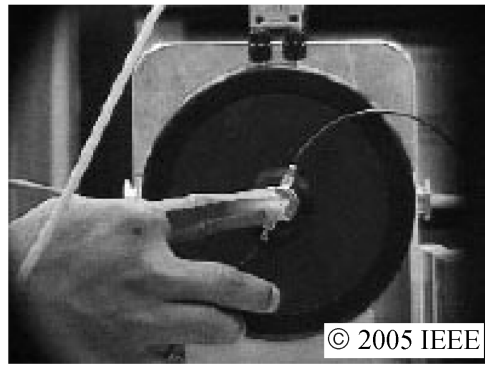

(d) Fu and Oliver (2005)

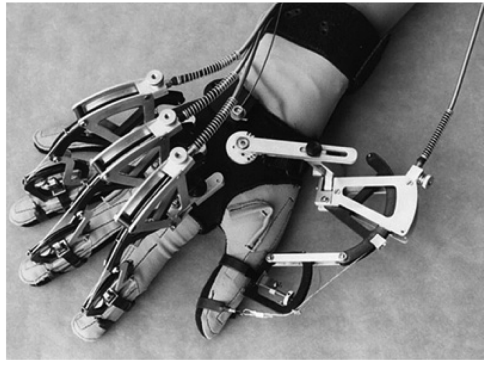

(e) Friedman and Flash (2007)

Figure 1. Some devices for finger stiffness measurement. Figures (a) through (d) are (c) IEEE, reproduced with permission. Figure (e) shows the CyberGrasp haptic glove, photo courtesy of CyberGlove systems LLC, reproduced with permission.

the stiffness at the finger tip, that is the stiffness of the whole finger linkage ${ }^{1}$ (see Milner and Franklin 1998). But, all in all, most of them have been constrained about a single joint, namely, the DIP joint in the case of the thumb or the MCP joint in the case of the index finger. This choice is justified by studies on grasping kinematics and force distribution among different fingers during object manipulation that demonstrated the existence of different force distributions and coordination schemes such as enslaving and force sharing (Friedman and Flash 2007).

The aim of the present study is the design of a device to perform measurements of the stiffness of the thumb and of the index finger during flexion and extension movements. As a matter of fact, these two movements predominate in many finger actions, e.g. grasping, pinching, pushing, etc.

This paper presents the preliminary experiments, the design choices and the simulations that guided the design

\footnotetext{
${ }^{1}$ The finger consisted of three joints, the distal interphalangeal (DIP), proximal interphalangeal (PIP) and metacarpophalangeal (MCP) joints (Kendall et al. 2005).
}

of a 2-finger exoskeleton. Using only two torque motors, the proposed device over-imposes a controlled displacement of the MCP joint of the finger in one of the two directions in the plane defined by flexion and extension of the finger joints and measures:

- The angular displacement resulting from this perturbation

- The interaction force between the device linkage and the first phalanx of the finger

Moreover, the results of some initial experiments have been reported in Section 5.

\section{Concepts}

\subsection{The hand complex}

The human hand is a complex mechanical device: it has a multi-degree-of-freedom structure (four major ones per finger, which are sometimes coupled with less important movements) actuated with hierarchical synergies. 
Moreover, every finger is controlled by two sets of muscles: the extrinsic muscles, which exert primary force, and the intrinsic muscles, which serve coordinating and stabilising functions. Almost all of these muscles are bi- or tri-articular (Tubiana 1981; Levangie and Norkin 2005).

The geometrical arrangement of some muscles is such that they flex one joint while extending another. Consequently, co-contraction of finger flexor and extensor muscles occurs frequently. As a result of these simultaneous co-contractions, the finger exhibits a relation between joint stiffness and joint torque that is more complex than the arm's.

Because of this complexity, an interesting convention has been proposed within the framework of virtual reality (VR) and haptic interfaces (Stergiopoulos et al. 2003). It consists of classifying the tasks executed with the hand in three categories:

- Tasks that require the use of only one finger (i.e. pressing a button, feeling the surface of an object, etc.)

- Tasks that can be done either with a minimum of two fingers or with the thumb and the rest of the fingers working together (i.e. pulling a lever, pushing objects, etc.)

- Tasks that require dexterous manipulation (i.e. holding an object with the fingers, turning a radio button, etc.), which require the simultaneous use of at least three fingers

Even though the aim of the present study is substantially different from the design of an haptic device for VR, we will consider the same classification and focus on the tasks that can be done either with a minimum of two fingers or with the thumb and the rest of the fingers working together. In both the cases mentioned above, we will measure only the stiffness of the thumb and of the index finger.

\subsection{Grip range of force}

To identify the design specifications of the new hand exoskeleton device, it was necessary to acquire a base knowledge about the interaction forces that the hand exerts on the object that it is manipulating.

The first experiment involved the measurement of the automatic increase of the grip force in order to prevent a grasped object from sliding when its weight changes (Figure 2).

The experiment was aimed at identifying the force which a subject exerts on the object that he is lifting when the object tends to slip downwards because of its weight and texture.

These measured values, which are much lower than the maximum force that a grasp can perform, were helpful in identifying the range of forces that our device will be subject to.

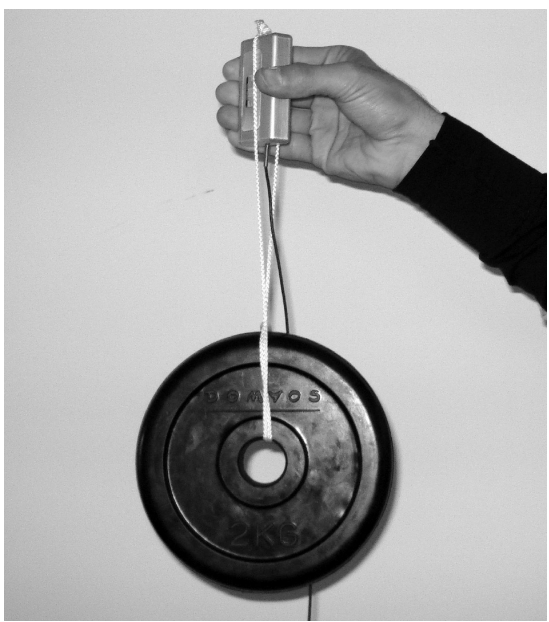

Figure 2. A preliminary experiment had the aim of measuring how grasp force is automatically increased to prevent a grasped object from sliding when the object is pulled down by an external force.

In order to have an approximate idea of the minimal requirements for actuators that would have been mounted on the exoskeleton and choose the parts that could really fulfil the task, a short preliminary experiment was prepared and executed by one subject in two different conditions:

- Letting him look at the hand-gripper and at the measured force shown on a laptop screen

- Forcing him to look elsewhere

Two different conditions were considered and the highest values were taken, as the intensity with which the visual feedback could affect the strength of the grip was unknown.

The set-up was made by a FUTEK LMD500 handgripper static force sensor ${ }^{2}$ to measure the force used to prevent the grasped object from slipping, a standard National Instruments data acquisition board (NI-USB6211) to get the data and a custom LabView interface to plot and store them. The results of this test are presented in Table 1. They obviously do not represent average values for an ideally large mixed group of population, but anyway give a good initial indication of the necessary force range.

Because of friction changes, the aforementioned values can be dramatically influenced by the texture of the surface of the grasped object.

\subsection{The spinal stretch reflex}

The third really important performance agent that was considered during the choice of the actuators and of the transmission mechanisms was the dynamics of the motion.

${ }^{2}$ Futek LMD500 Medical Load Cell (Hand). Available from: http://www.futek.com/product.aspx?stock=FSH00125. 
Table 1. Data collected from the experiment shown in Figure 2. The measured values are lower than the $15 \%$ of the force that a hand can exert.

\begin{tabular}{lcc}
\hline Hanging weight $(\mathrm{kg})$ & Grip force $^{\mathrm{a}}(\mathrm{kg})$ & Grip force $^{\mathrm{b}}(\mathrm{kg})$ \\
\hline 0.5 & 0.6 & 0.8 \\
1.0 & 1.1 & 1.3 \\
1.5 & 1.8 & 2.2 \\
2.0 & 2.4 & 4.2 \\
2.5 & 3.3 & 5.0 \\
3.0 & 4.3 & 6.5 \\
3.5 & 4.7 & 7.8 \\
\hline
\end{tabular}

${ }^{\mathrm{a}}$ The subject was given a visual feedback.

${ }^{\mathrm{b}}$ The subject was not given a visual feedback.

Speed and acceleration profiles had to be taken into account in order to grant that the over-imposed perturbation would happen before the subject could be aware of what was happening and react to the stimulus. The reaction that the subject is expected to have whenever her/his limbs are moved by an unexpected external perturbation is called spinal stretch reflex.

In medical literature, a spinal stretch reflex is defined as a muscle contraction performed as a response to stretching within the muscle. When the activated muscle is stretched by an outside agent, it contracts more forcefully than before the stretch, as if it were making an extra effort to regain its original length.

The traditional explanation of the stretch reflex is that when the whole muscle is stretched, the spindle organs are stretched by the same relative amount, since they are mechanically in parallel with the muscle. The stretch of the spindles is sensed by the type $\mathrm{I} a$ afferents, which cause an increase in the firing rate of the alpha motoneurons controlling the force level in the muscle. A small stretch, which might have been responsible for only a small rise in force in an isolated muscle with fixed $\alpha$ activity, can be responsible for a large rise in force when the reflex loop is intact. When the physician strikes the patellar tendon over the patient's knee, he produces a small stretch of the quadriceps group of muscles, followed by the reflex kick of the quadriceps which tells that the nervous system wiring is all in good order. The stretch reflex is fast because it involves only one synapse, that between the axon of the I $a$ afferent and the motor neuron.

Several studies investigated the propagation time of the stimulus and the time that is required to react to it both in the upper and in the lower limbs. Even if it is known that in healthy subjects the responsiveness of the stretch reflex is modulated during human voluntary limb movements (Milner and Franklin 1998), the goal of this work was the design of a device that can perform transients with a maximum duration of $30 \mathrm{~ms}$. This choice should ensure the total absence of reaction in most of the cases (Hajian and Howe 1997).
These three main requirements led our choices and were considered as rigid parameters in the feasibility study as well as in the preliminary mechanical simulations.

\section{Device design}

The development of the device that this study is proposing for measuring the stiffness of the finger can be divided into four different phases that have been carried out subsequently. These phases concerned

- the mechanical design of all the parts and the structural and kinematic analysis of the linkage;

- the analysis of the dynamics of the exoskeleton and of the interaction between the device and the finger; this study mainly concerns the torque and speed transmission coefficient from the motor shaft to the finger MCP joint;

- the synthesis of the minimal requirements for the actuators and the choice of the motors that better could fulfil the task;

- the choice of the on-board sensors necessary to measure the stiffness components.

\subsection{Structural design}

Concerning the first phase of the project, the initial decision was to make a device that could over-impose a perturbation only on the MCP joint of the thumb and of the index finger. The aim of this preliminary choice was to reduce at most the number of actuators, make the device as less invasive as possible, remove everything that could make the task too unnatural for the subject and finally avoid every unnecessary mechanical coupling between the device parts and the finger.

Then, in order to diminish complexity and weight at the same time, we chose to design a rigid 3-link directdriven under-actuated serial mechanism. This kind of direct transmission is essential to avoid the continuous control of cable tensioning, which is necessary in devices that use cable transmissions, and the introduction of different stiffness components (i.e. the cable). The compliance usually presented in cable transmission systems is an undesirable feature for the applications of the proposed device since it could affect the finger stiffness measurement.

The single motor used for each of the 3-link chains actuates the proximal link, while the middle and the distal links work as force transmission elements. The motors are mounted on a base at the back of the palm and are able to rotate around the vertical axis on ball bearings to allow finger abduction. Back-drivability of each of the 3-link chains is achieved with the use of a low reduction gearbox (14:1 reduction ratio) for the motor. Previous studies on haptic devices employing gear transmission have proven that small reduction ratios like the one chosen for this device do not affect the subject's final feedback in an important manner (see Longnion et al. 2001; Stergiopoulos et al. 2003). 


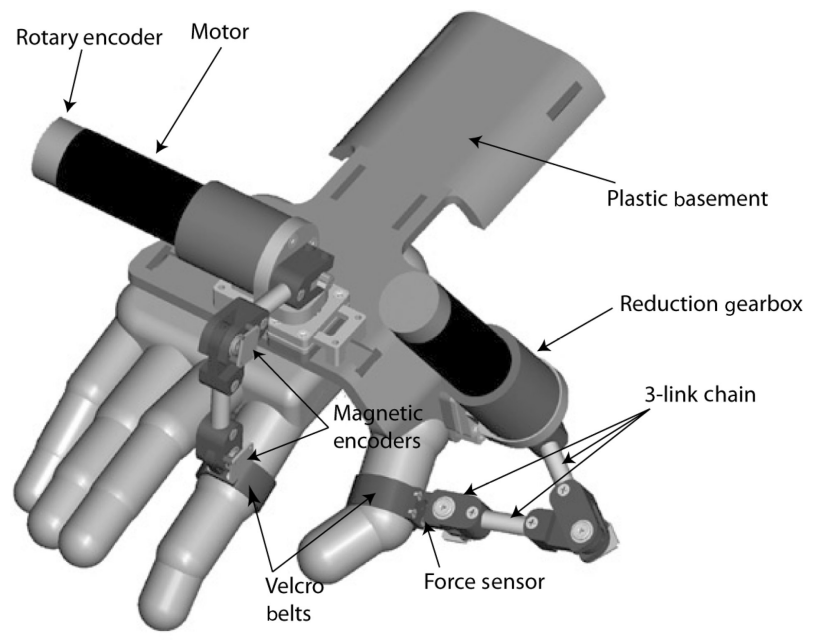

Figure 3. Exoskeleton design: the indication text shows the main parts of the device.

The assembled structure (consisting of linkages, actuators, sensors, etc.) was conceived as fixed on the dorsal plate of the hand: this solution allows the user to manipulate objects without having mechanical parts that could impede the movement of the fingers and their interaction with the grasped object, or even affect the posture of the grasp itself.

This choice was also helpful to better sustain the exoskeleton weight, which is considerable (mainly because of the motors) and might cause fatigue to the user. For compensating this load, the device is fastened not only to the dorsal plate of the hand, but also to the wrist and to the most distal part of the forearm (see Figure 9).

The design of the mechanical structure of the system

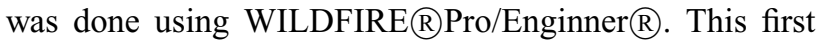
phase ended with the development of a complete 3D model. A snapshot of the model is shown in Figure 3.

\subsection{Mechanical simulations}

Having designed the basic mechanical configuration of the device, a set of simulations involving the main static and dynamic components of the motion were carried out in order to estimate interaction forces and examine the behaviour of the system, when coupled with the hand complex. These studies gave us both a measure of the torque propagation from the motor shaft to the MCP joint and valuable data to choose the best compromise between weight, size and torque for the motors.

The tool used to analyse the kinematic and dynamic aspects of the mechanical device and its coupling with a human finger was the SimMechanics ${ }^{\mathrm{TM}}$ toolbox for Matlab $\AA$. The exoskeleton linkage and the finger were modelled using the weight and the inertia data respectively taken from the pro/engineering model (see Table 2 ) and empirically obtained from anthropometric measurements performed in previous studies concerning hand tools ergonomics and robotics (Nag et al. 2003; Veber and Bajd 2006). Then two sets of simulation were implemented.

The first set of simulations consisted of static simulations (see Figure 4) that were carried out to estimate how much force is transmitted from the motor shaft to the finger MCP joint. The finger MCP joint angle was blocked in a rest position and a torque of $0.08 \mathrm{~N} / \mathrm{m}$ was applied on the motor shaft.

Observing the results of the simulation it can be estimated that, in the rest joint configuration shown in Figure 5, $\mathrm{MCP}_{\text {torque }} \simeq 0.6 \times$ Motor $_{\text {torque }}$.

The second set of simulations concerned the analysis of the dynamics of the device when fastened to the hand and connected to the finger. The aim of these studies was to observe the torque required by the motor in order to reach a desired displacement of $0.09 \mathrm{rad}$ from the starting position in about $20 \mathrm{~ms}$.

As considered in Section 2, transients with a maximum duration of $20 \mathrm{~ms}$ were used to ensure that the data were

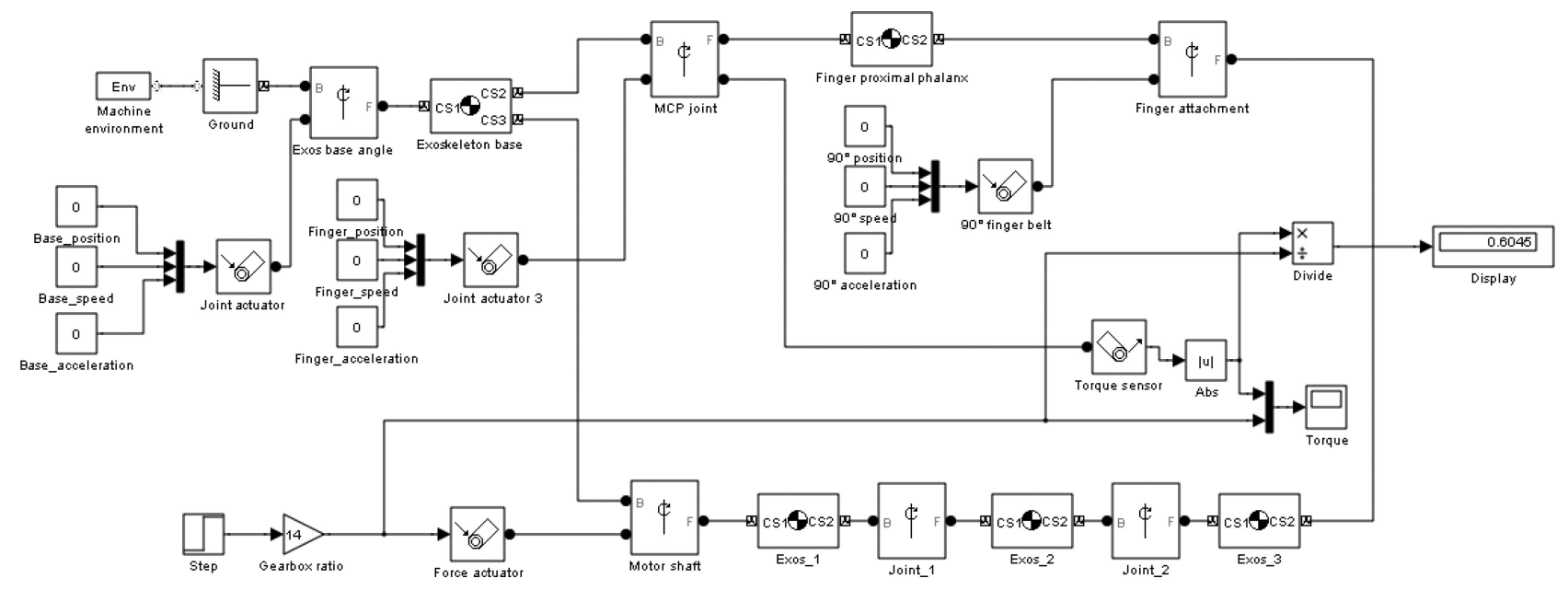

Figure 4. Static simulation scheme. 
Table 2. Weight and inertia data of the finger and exoskeleton linkage.

\begin{tabular}{|c|c|c|c|c|}
\hline & Mass (kg) & Length (m) & \multicolumn{2}{|c|}{ Inertia matrix $\left(\mathrm{kg} \mathrm{m}^{2}\right)$} \\
\hline Finger $^{a}$ & 0.025 & 0.04 & $\left(\begin{array}{cl}2.0 \times 10^{-7} & 0 \\
0 & 5.0 \times 10^{-6} \\
0 & 0\end{array}\right.$ & $\begin{array}{r}0 \\
0 \\
5.0 \times 10^{-6}\end{array}$ \\
\hline Exoskeleton proximal link & 0.1 & 0.056 & $\left(\begin{array}{cl}1.0 \times 10^{-7} & 0 \\
0 & 1.5 \times 10^{-5} \\
0 & 0\end{array}\right.$ & $\begin{array}{r}0 \\
0 \\
1.5 \times 10^{-5}\end{array}$ \\
\hline Exoskeleton intermediate link & 0.1 & 0.058 & $\left(\begin{array}{cl}1.0 \times 10^{-7} & 0 \\
0 & 2.0 \times 10^{-5} \\
0 & 0\end{array}\right.$ & $\begin{array}{r}0 \\
0 \\
2.0 \times 10^{-5}\end{array}$ \\
\hline Exoskeleton distal link & 0.1 & 0.017 & $\left(\begin{array}{cl}1.0 \times 10^{-7} & 0 \\
0 & 2.0 \times 10^{-6} \\
0 & 0\end{array}\right.$ & $\begin{array}{r}0 \\
0 \\
2.0 \times 10^{-6}\end{array}$ \\
\hline
\end{tabular}

${ }^{\mathrm{a}}$ The reported length is the length of the first phalanx while the inertia is the whole finger one.

collected before the occurrence of complicating contributions due to either the stretch reflex (responding to muscle change), at approximately $30 \mathrm{~ms}$ following the onset of a transient, or to longer latency responses such as the cutaneous slip reflex or voluntary muscle contraction. Disallowing these muscular contributions permits identification of the impedance of the MCP joint independent of any sensory feedback loops. The simulation scheme is shown in Figure 6.

The position control signal is a step that has been conveniently smoothed to simulate a real transient of the signal and given to a PID controller; the PID parameters found

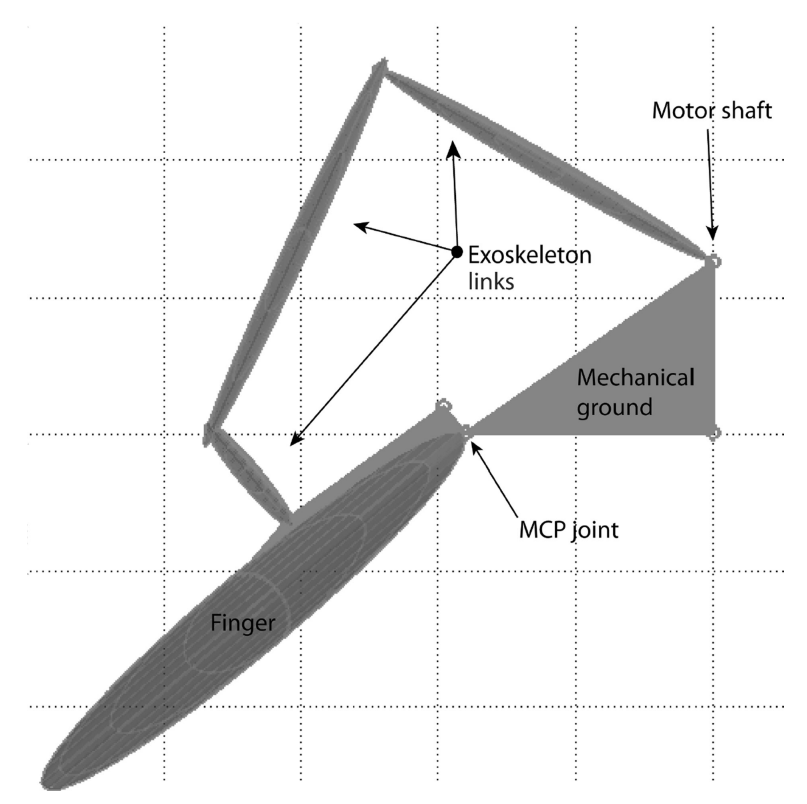

Figure 5. Extended finger and exoskeleton linkage Sim Mechanics ${ }^{\mathrm{TM}}$ models. empirically for the simulation model were also used during the experimental recordings as starting point to tune the exoskeleton control board's PID parameters.

The results of these simulations gave us some indications about the minimal requirements of the motor in terms of torque and speed.

Figure 7 shows the MCP joint angular position: it reaches the desired position $(0.09 \mathrm{rad})$ in about $24 \mathrm{~ms}$ as required for the task.

Figure 8 displays the following:

- The couples (Torque, Speed) that the motor has to realise in order to follow the reference position trajectory

- The nM-curve of the chosen motor

The simulations made us opt for two Maxon RE $25 \phi$ $25 \mathrm{~mm}$, graphite brushes, 20 Watt brushed DC motors ${ }^{3}$, assembled with a 14:1 ceramic planetary gearbox and a $1000 \mathrm{CPT}^{4}$, three-channel rotary optical encoder. These motors can exert (even without the gearbox) a torque up to $257 \mathrm{mNm}$ (stall torque) and have a nominal speed of 8360 rpm. The nM-curve computed from these values is plotted in Figure 8.

\section{Device construction}

The prototypes of the 3-link chains were made mainly in aluminium with only some joint sections (shafts) fabricated in steel. The support base was built in ABS-plastic at the IIT laboratories using a high-tech $3 \mathrm{D}$ printer.

\footnotetext{
${ }^{3}$ Maxon RE 25 Brushed Motors. Available from: http://shop. maxonmotor.com/ishop/article/article/118752.xml.

${ }^{4}$ Counts Per Turn - this parameter specifies the resolution of the encoder.
} 


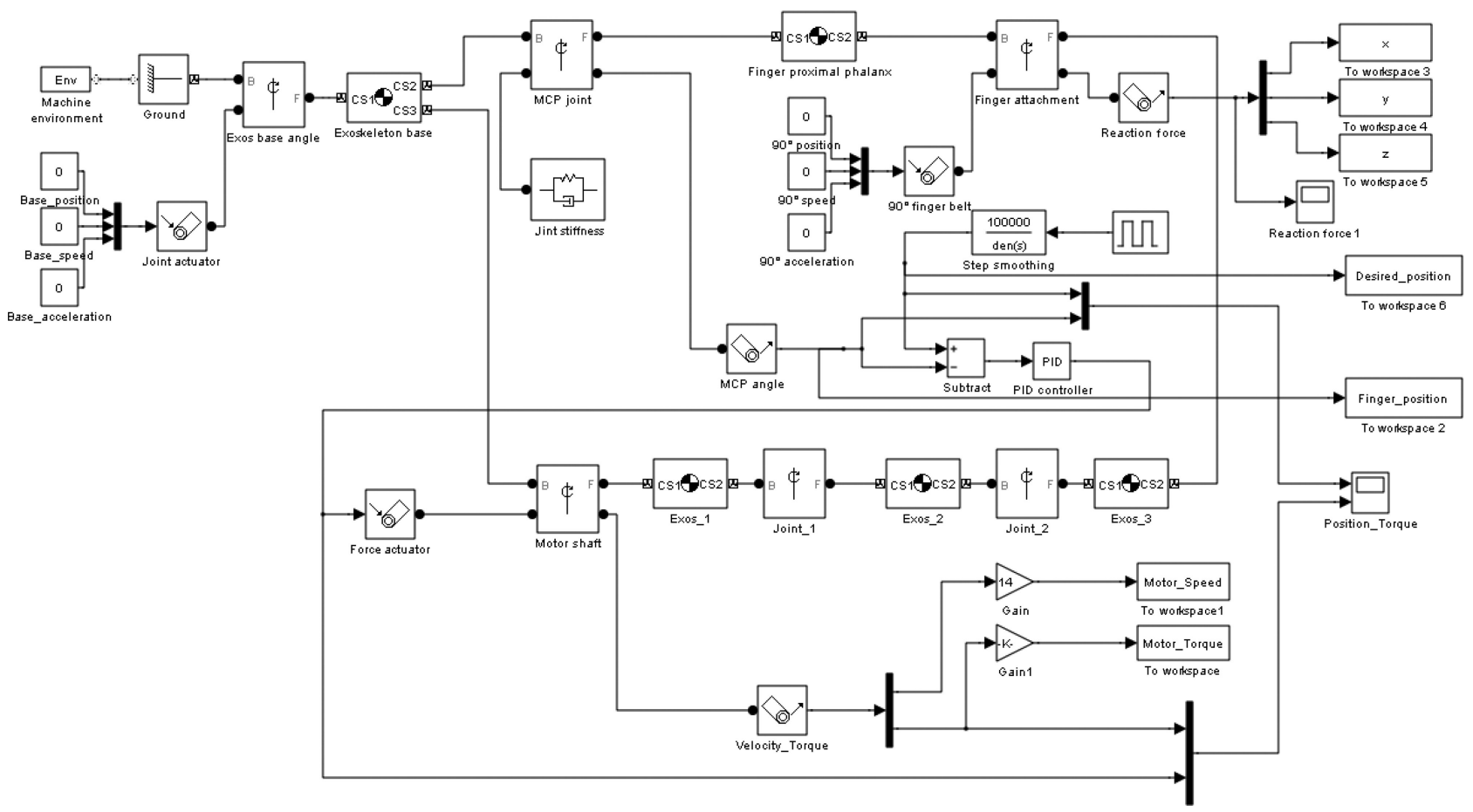

Figure 6. Dynamic simulation scheme.

ABS-plastic is a common thermoplastic used to make light, rigid, moulded products such as piping, automotive body parts and protective head gear.

A picture of the first prototype device is shown in Figure 9.

\subsection{Force sensors}

Physics concepts define the stiffness as the resistance of an elastic body to deflection or deformation by an applied

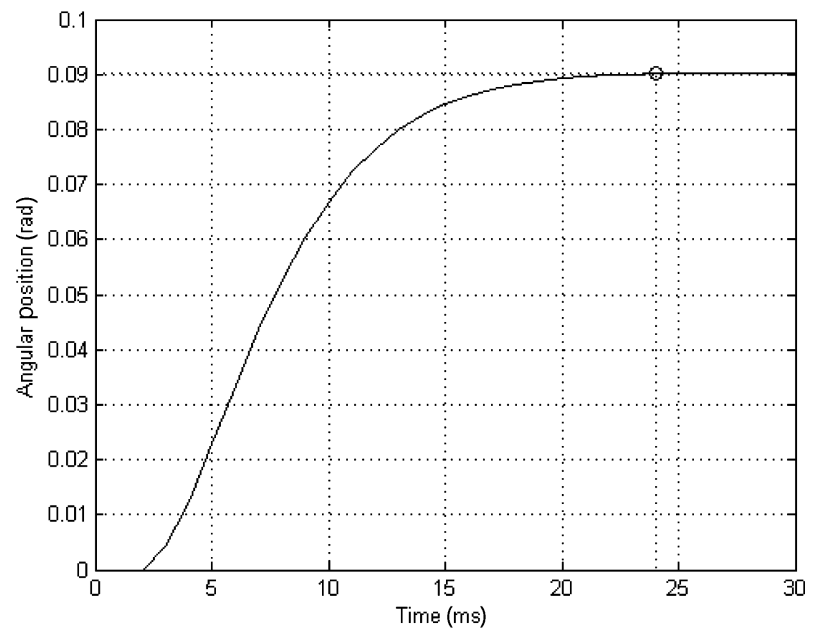

Figure 7. MCP joint angular position: it reaches the desired displacement $(0.09 \mathrm{rad})$ in about $24 \mathrm{~ms}$. force. The stiffness, $k$, of a body is defined as $k=P / \delta$, where $P$ is the applied force expressed in Newtons and $\delta$ is the deflected distance expressed in metres (i.e. the stiffness is measured in $\mathrm{N} \mathrm{m}^{-1}$ (newtons per metre)).

In order to measure these two quantities, two different kinds of sensors have been mounted on the device: force sensors and position sensors.

To enable the finger exoskeleton with the capacity to measure the interaction forces with the finger segment,

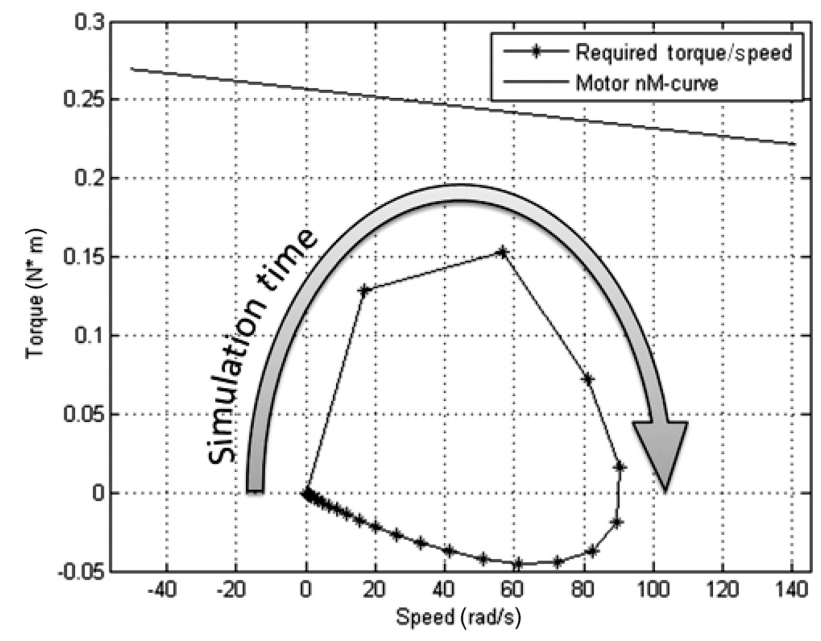

Figure 8. Motor nM-curve and Torque-Speed trajectory required for the task execution. All measurements are referred to the motor shaft (w/o gearbox). 

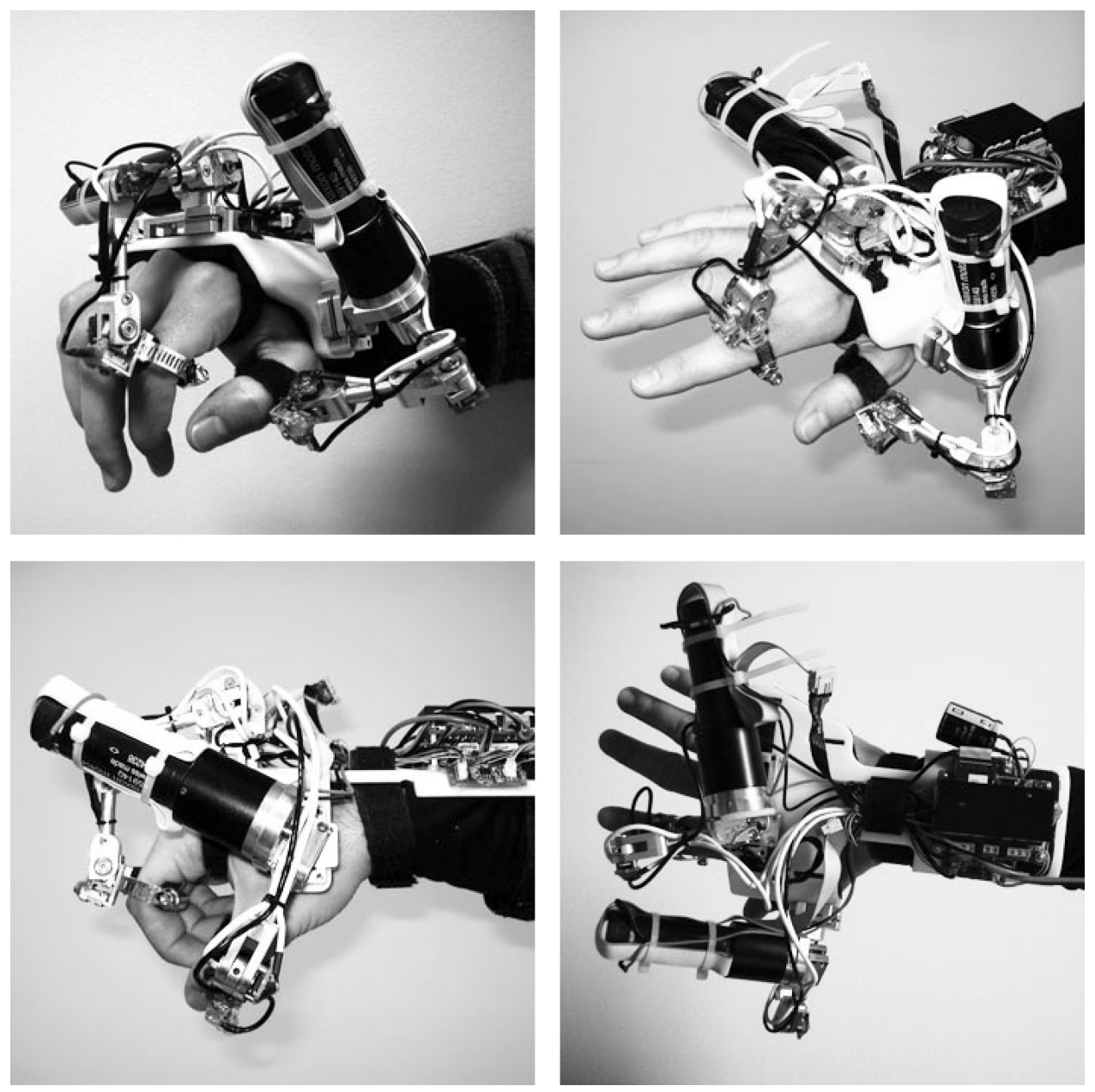

Figure 9. The 2-finger hand exoskeleton.

a force sensor was integrated into the exoskeleton chain. Commercial force sensors are both expensive and most of the times not mechanically compatible (size, mechanical interface) with small robotic systems such as the finger exoskeleton. Therefore, it was chosen to design our own custom-made force sensor. They constitute the proximal links of the exoskeleton, the ones that are fastened to the fingers.

Both the load cells and the electronics were designed inhouse for the purpose of dimensional optimisation. The load cell is based on an ' $\mathrm{S}$ '-type beam which is machined from a solid aluminium block to reduce hysteresis and increase the strength and repeatability.

Semiconductor strain ganges placed on the sides of the beam structure allow the measurement of the strain generated on the beam when a load is applied. Finite element stress/strain analysis was used to optimise the selection of the strain gauge position as well as the dimensioning of the load cell structure. Typical results of such a study are shown in Figure 10, which shows the strain curve along the side of the ' $\mathrm{S}$ ' beam. Based on these, the position of the strain gauge elements was fixed to $5 \mathrm{~mm}$ from the edge.

Moreover, as shown in the assembled CAD design (Figure 11a), this part of the linkage is attached to another L-shaped part so as to ease the finger fixation and improve the sensitivity of the sensor.

The strain gauges of each beam are connected in an half-bridge electrical configuration and the signal coming from the load cell is then amplified and routed into the control board analogue channels.

The load cell and a magnification of the strain-gauge part are depicted in Figure 11b.

\subsection{Position sensors}

To measure the position of the 3-link joints, a 12-bit programmable magnetic rotary encoder was mounted at each joint. The encoder consists of two parts:

- A magnet, that is mounted inside the rotating part of the joint. 


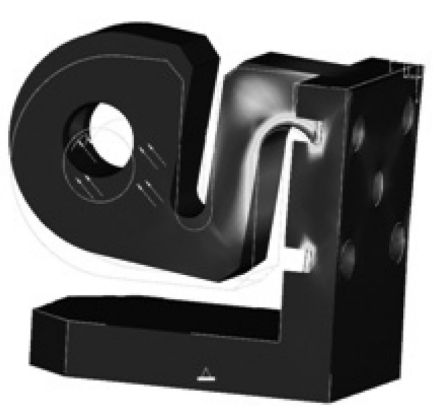

(a)

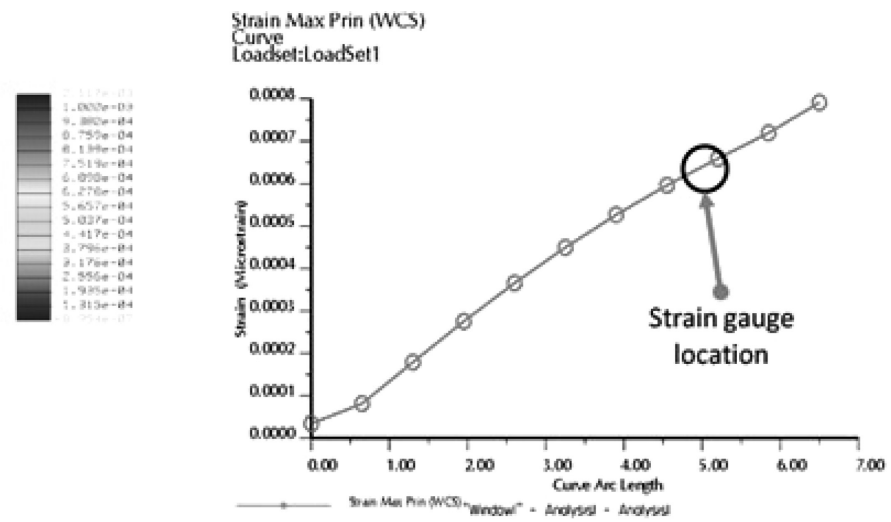

(b)

Figure 10. Strain generated on force sensor when a load is applied to the load cell - load parameters $\left(40 \mathrm{~N}\right.$ at $45^{\circ}$ from the horizontal plane).

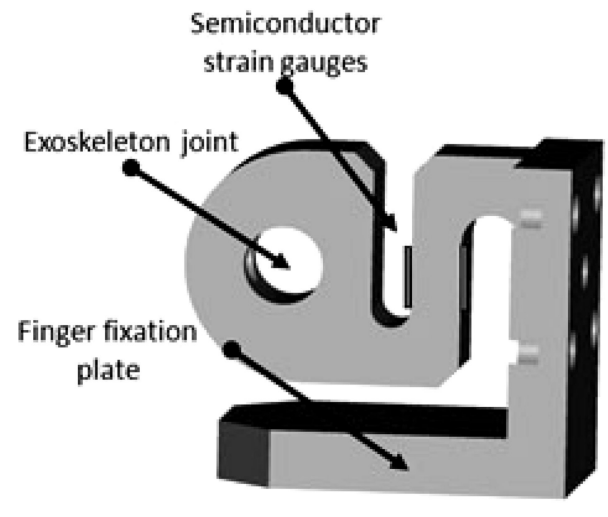

(a) Sensor CAD design

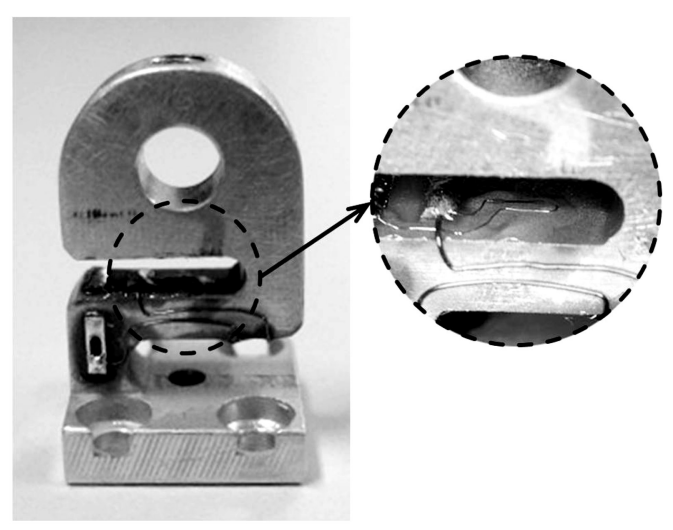

(b) A sensor detail

Figure 11. Custom force sensor.

- An AS5045 sensor ${ }^{5}$, attached to the fixed part of the joint. 12.

The magnetic encoder integration can be seen in Figure

\subsection{Distributed control system}

Another part of the device development was the choice of the proper control system. In our case, the two fingers' position and force are controlled using a distributed control system made of

- a laptop computer that will define the high-level commands and

\footnotetext{
${ }^{5}$ Austriamicrosystems AS5045 12 Bit Programmable Magnetic Rotary Encoder. Available from www.austriamicrosystems.com.
}

- a low-level controller to execute these commands.

The laptop computer runs a 'client' application that has been developed integrating code programmed in Microsoft Visual $\mathrm{C}++$ and in National Instruments LabView ${ }^{\mathrm{TM}}$. The aim of these program is to

- initialise the communication protocol between the computer and the exoskeleton's on-board controller;

- set many of the controller parameters;

- calibrate and initialise the encoder positions;

- generate the over-imposed finger relative displacements and the time of occurrence of the stimuli;

- reconstruct the finger's MCP angle from the encoders position (the kinematic model of the finger joints is bundled inside the program);

- synchronously monitor, acquire and store the data of position and force. 


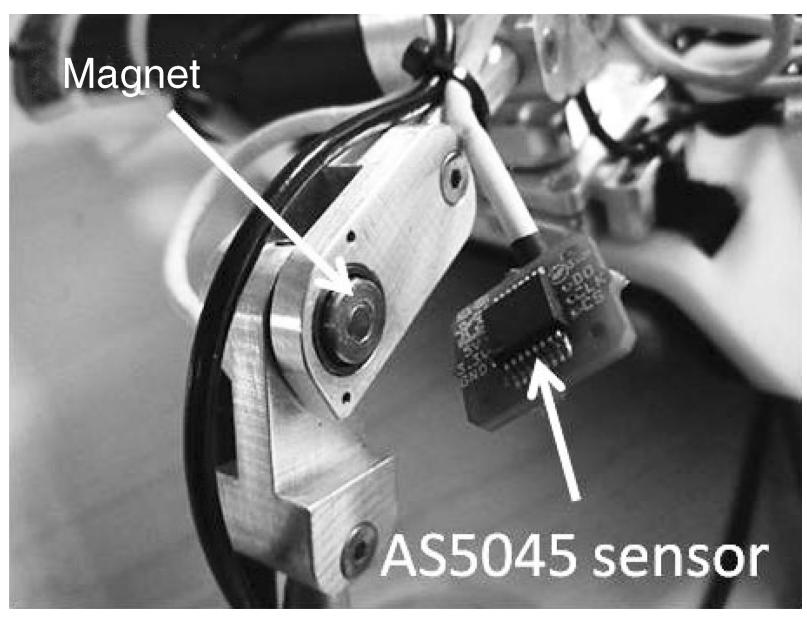

Figure 12. A magnetic encoder sensor composition detail.

The on-board controller ${ }^{6}$, which is based on a Freescale 56F807 DSP and is made of two components (logic and power), is connected to the software running on the laptop through a CAN-bus adapter ${ }^{7}$ and acts like a 'server' device.

Each control board

- decodes the command messages coming from the PC side;

- generates the velocity profiles from the actual position to the desired position;

- controls the brushed-DC motors;

- reads the encoders' and the sensors' measurements and sends the acquired value and the state reporting messages of the board as periodic messages through the CAN-bus.

The control board can deliver a DC current of $6 \mathrm{~A}$ at $48 \mathrm{~V}$ and, in the present firmware implementation, is programmed to return a complete set of messages every $4 \mathrm{~ms}$ ( 250 samples per second). The rate of message broadcasting will be increased in the future firmware implementations in order to produce a bigger and more detailed amount of data.

At present, the firmware running on the board implements a closed-loop position control on the motor shaft. In future, according to the specific experiment requirements,

\footnotetext{
${ }^{6}$ The control board and the magnetic encoder board have been developed as part of the project 'RobotCub: European Commission Cognition Unit, Project no. IST-2004-004370' (see Metta et al. 2008; RobotCub 2008).

${ }^{7}$ Controller-area network (CAN or CAN-bus) is a computer network protocol and bus standard officially released in 1986 at the SAE (Society of Automotive Engineers) congress and designed to allow micro-controllers and devices to communicate with each other without a host computer. It was designed specifically for automotive applications but is now also used in other areas.
}

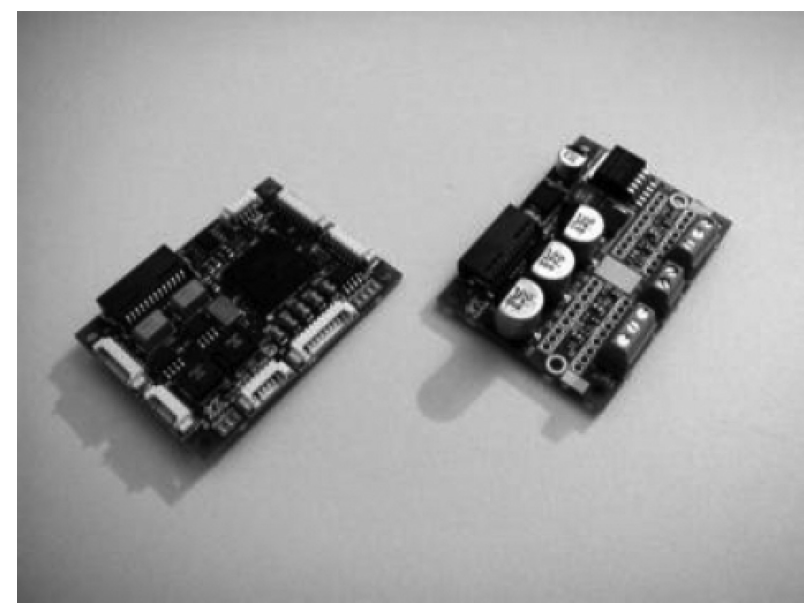

Figure 13. The brushed-DC motor control logic and power amplifier of the exoskeleton. The size of the two PCBs is $58 \times$ $42 \mathrm{~mm}$.

it could be modified to fulfil our specific needs by closing the control loop on the reconstructed position of the MCP angle or on the data coming from the force sensor.

\section{Experimental protocol}

In the first experimental set-up, the subject wears the exoskeleton and keeps his arm relaxed and horizontally leaned on a desk while his index finger is perturbed 30 times with a fixed value displacement (1000 encoder pulses). The delay between each perturbation and the next is randomly chosen by the computer control software. This way the subject is not able to predict the stimulus occurrence.

After having initialised the control parameters, the control loop executes the following:

- Enables the motor control;

- Reads the actual position of the finger;

- Computes the goal position by adding the displacement value to the actual position;

- Verifies whether the goal position falls inside the allowed range of movement - the device is equipped with mechanical stops in order to prevent injuries;

- Commands the DSP board to move the motor at near maximum speed to the desired position or, if the computed position has been truncated, to the motion threshold;

- Starts reading and storing all the force and position broadcast messages from the beginning of the movement to the desired position arrival;

- Saves all the data to an ASCII measurement file;

- Releases the motor - so the finger can freely come back to its rest position;

- Waits for a random delay. 

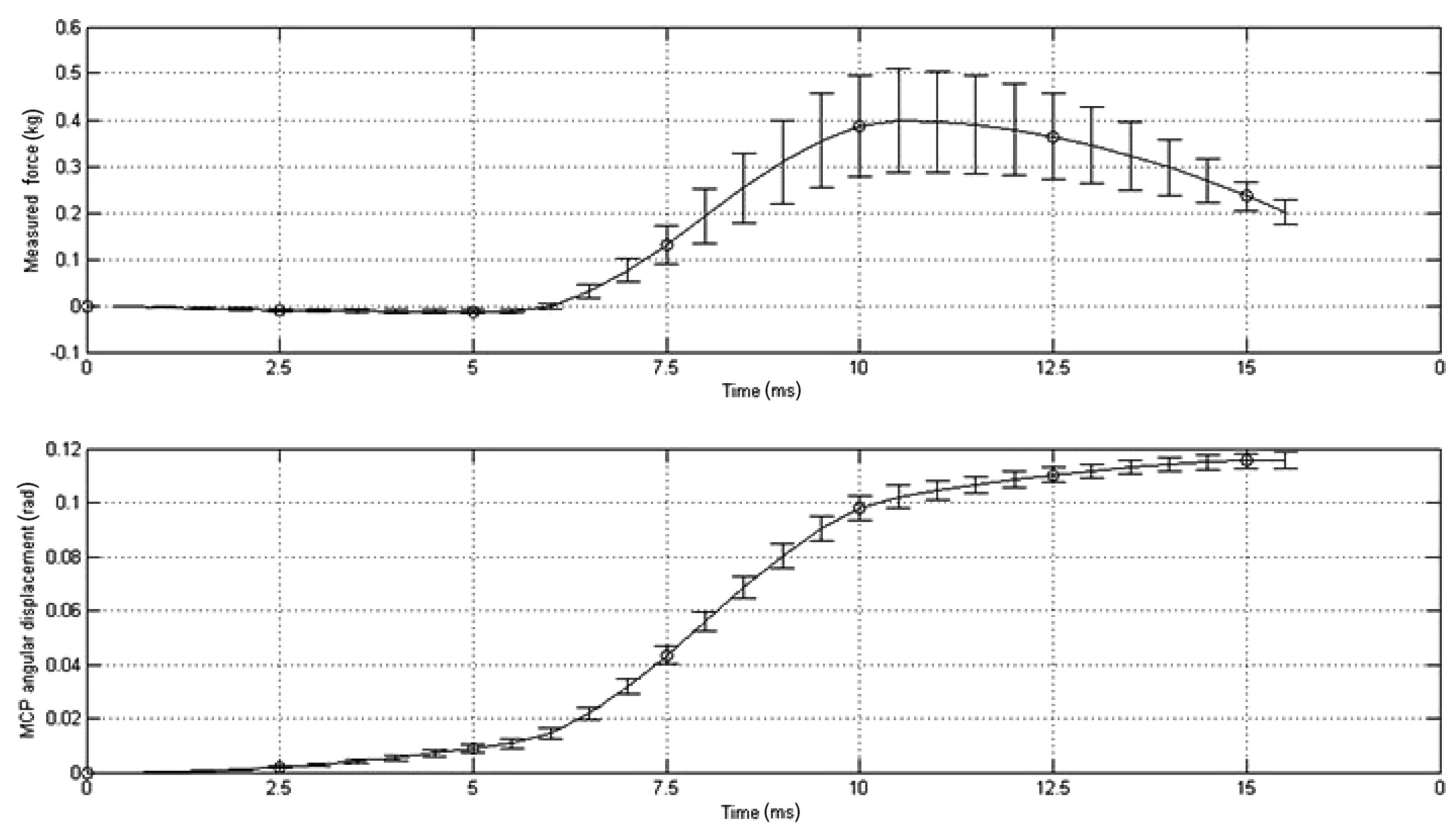

Figure 14. Experimental data. The plot shows the mean and the standard deviation of the acquired force and MCP joint angle.

An interpolated plot of the gathered data is presented in Figure 14. It shows the force measured by the strain sensor and the reconstructed angular position of the MCP joint.

\section{Discussions}

Mechanical coupling between the exoskeleton and the human hand makes the behaviour of the device almost unpredictable while applying a perturbation on the finger. Moreover, most of the parameters concerning the finger dynamics have not yet been estimated. Our goal was to build an exoskeleton that could be a good compromise between power, speed, wearability and weight.

Some initial simulations gave us a few guidelines about how to design the device's kinematics and choose the proper motors and reduction gearboxes.

At present, first initial tests have proven that the device is wearable and light enough and also that it can perturb the hand in the desired way, even though motor inertia can affect abduction/adduction in certain orientations of the hand during he reach-to-grasp movement.

\section{Conclusions and future work}

Several studies have proven that the impedance properties determine the stability of the grasp and must be appropriately selected and tuned in order to successfully perform manipulation tasks. The analysis of the human finger stiffness during grasping and interaction exercises is useful both for investigating the human control of stiffness and for introducing dexterous manipulation in robotic humanoid hands.

The initial aim of this study has been the design of a 2finger hand exoskeleton for finger stiffness measurements.
Our final goal will be to measure the stiffness of the fingers during the reach-to-grasp movement in order to understand whether the human brain somehow prepares the hand for the grasp. In the future experiments, the subject will be asked to perform several trials of a reaching task. During these trials, he will move his hand from a rest position towards the object that he is asked to grasp. For the whole duration of the experiment, the relative distance between the fingertip and the contact point on the object will be measured using a motion capture system.

In the upcoming experiments, the program running on the computer will define the trials during which the subject's hand will be perturbed, the instant of triggering and the intensity of the perturbation. This way, the subject would not be able to predict in which trials, and when during the trial, the perturbation will occur. In any case, the hand will always be perturbed before it collides with the object. Doing so, we can measure the stiffness of the finger only, instead of the interaction forces between the finger and the object.

Using this information, our target is to synthesise effective models of hand stiffness control in manipulation tasks for humanoid robots.

Moreover, our intention is also to test the real advantages of a device mounted entirely on the back of the hand. This further phase of the project could include the displacement of the motors on a holder fastened to the forearm and the introduction of cable transmissions. With this modified design, the weight of the motors will not put burden on the hand and the wrist anymore, and it will be better balanced during the task execution. In that case, the motor encoder would be replaced with an incremental encoder mounted on a pulley so that the good quality of the data would be preserved, holding off the stiffness of the metal cable. 
Finally, the device, which is now at the end of a test phase, would hopefully be a powerful platform even for various physiological hand measurements. The communication interface of this device (CAN-bus) will allow the easy integration with a variety of different hardware and software packages, both commercial and custom made, as motion capture system, electromyography (EMG) acquisition devices, virtual reality and tele-operation environments.

\section{Acknowledgements}

We thank Gabriel Baud-Bovy of S. Raffaele Medical Center, Milano, Italy, for a preliminary, really extensive, discussion of this research project and for his helpfulness during all the phases of the project. We also thank Prof. Thierry Pozzo, Prof. Darwin Caldwell, Marco Jacono and Lorenzo Masia of IIT, Genova, Italy, for their valuable advices on physiology, mechanics, computer-aided simulations and human impedance concepts.

Finally, we want to acknowledge the RobotCub consortium for having designed and maintained the development of the electronic devices included in this project.

\section{References}

Asada H, Asari Y. 1988. The direct teaching of tool manipulation skills via the impedance identification of human motions. Proceedingsof IEEE International Conference on Robotics and Automation, 1988. vol. 2. p. 1269-1274.

Bennett DJ, Hollerbach JM, Xu Y, Hunter IW. 1992. Time-varying stiffness of human elbow joint during cyclic voluntary movement. Exp Brain Res. 88(2):433-442.

Friedman J, Flash T. 2007. Task-dependent selection of grasp kinematics and stiffness in human object manipulation. Cortex. 43(3):444-460.

Fu CY, Oliver M. 2005. Direct measurement of index finger mechanical impedance at low force. First Joint Eurohaptics Conference, 2005 and Symposium on Haptic Interfaces for Virtual Environment and Teleoperator Systems, 2005. World Haptics 2005. p. 657-659.

Gribble PL, Mullin LI, Cothros N, Mattar A. 2003. Role of cocontraction in arm movement accuracy. J Neurophysiol. 89(5):2396-2405.

Hajian AZ, Howe RD. 1997. Identification of the mechanical impedance at the human finger tip. J Biomech Eng. 119(1):109114.

Hogan N. 1990. Mechanical impedance of single- and multiarticular systems. In: Winters J, Woo S, (eds.), Multiple muscle systems: biomechanics and movement organization. New York: Springer-Verlag. p. 149-164.
Hunter IW, Kearney RE. 1983. Invariance of ankle dynamic stiffness during fatiguing muscle contractions. J Biomech. 16(12):985-991.

Jones LA, Hunter IW. 1990. Influence of the mechanical properties of a manipulandum on human operator dynamics. 1. Elastic stiffness. Biol Cybern. 62(4):299-307.

Kao I, Cutkosky M, Johansson R. 1997. Robotic stiffness control and calibration as applied to human grasping tasks. IEEE Trans Robot Autom. 13(4):557-566.

Kearney RE, Hunter IW. 1990. System identification of human joint dynamics. Crit Rev Biomed Eng. 18(1):55-87.

Kendall FP, McCreary EK, Provance PG, Rodgers MM, Romani W. 2005. Muscles: testing and function, with posture and pain. Philadelphia, PA: Lippincott Williams \& Wilkins.

Latash ML. 1992. Independent control of joint stiffness in the framework of the equilibrium-point hypothesis. Biol Cybern. 67(4):377-384.

Levangie P, Norkin C. 2005. Joint structure and function: a comprehensive analysis. Philadelphia, PA: F. A. Davis Company. Chapter 9. p. 305-352.

Longnion J, Rosen J, Sinanan M, Hannaford B. 2001. Effects of geared motor characteristics on tactile perception of tissue stiffness. Stud Health Technol Inform. 81:286-292.

McMahon TA. 1984. Muscles, Reflexes, and Locomotion. Princeton, NJ: Princeton University Press.

Metta G, Sandini G, Vernon D, Natale L, Nori F. 2008. The iCub humanoid robot: an open platform for research in embodied cognition. Special session on EU-projects.

Milner TE. 2002. Adaptation to destabilizing dynamics by means of muscle cocontraction.. Exp Brain Res. 143(4):406-416.

Milner TE, Cloutier C. 1998. Damping of the wrist joint during voluntary movement. Exp Brain Res. 122(3):309-317.

Milner T, Franklin D. 1998. Characterization of multijoint finger stiffness: dependence on finger posture and force direction. IEEE Trans Biomed Eng. 45(11):1363-1375.

Mussa-Ivaldi FA, Hogan N, Bizzi E. 1985. Neural, mechanical, and geometric factors subserving arm posture in humans. J Neurosci. 5(10):2732-2743.

Nag A, Nag PK, Desai H. 2003. Hand anthropometry of Indian women. Indian J Med Res. 117:260-269.

RobotCub. 2008. RobotCub website. Available from: http://www.robotcub.org/

Serres SJD, Milner TE. 1991. Wrist muscle activation patterns and stiffness associated with stable and unstable mechanical loads. Exp Brain Res. 86(2):451-458.

Stergiopoulos P, Fuchs P, Laurgeau C. 2003. Design of a 2-finger hand exoskeleton for VR grasping simulation. Eurohaptics 2003. Dublin, Ireland.

Tubiana R. 1981. The hand. Philadelphia, PA: W.B. Saunders Company.

Veber M, Bajd T. 2006. Assessment of human hand kinematics. ICRA 2006. Proceedings of IEEE International Conference on Robotics and Automation, 2006. p. 2966-2971.

Whitney D. 1982. Quasi-static assembly of compliantly supported rigid parts. Trans ASME J Dyn Syst, Meas Control. 104:65-77. 

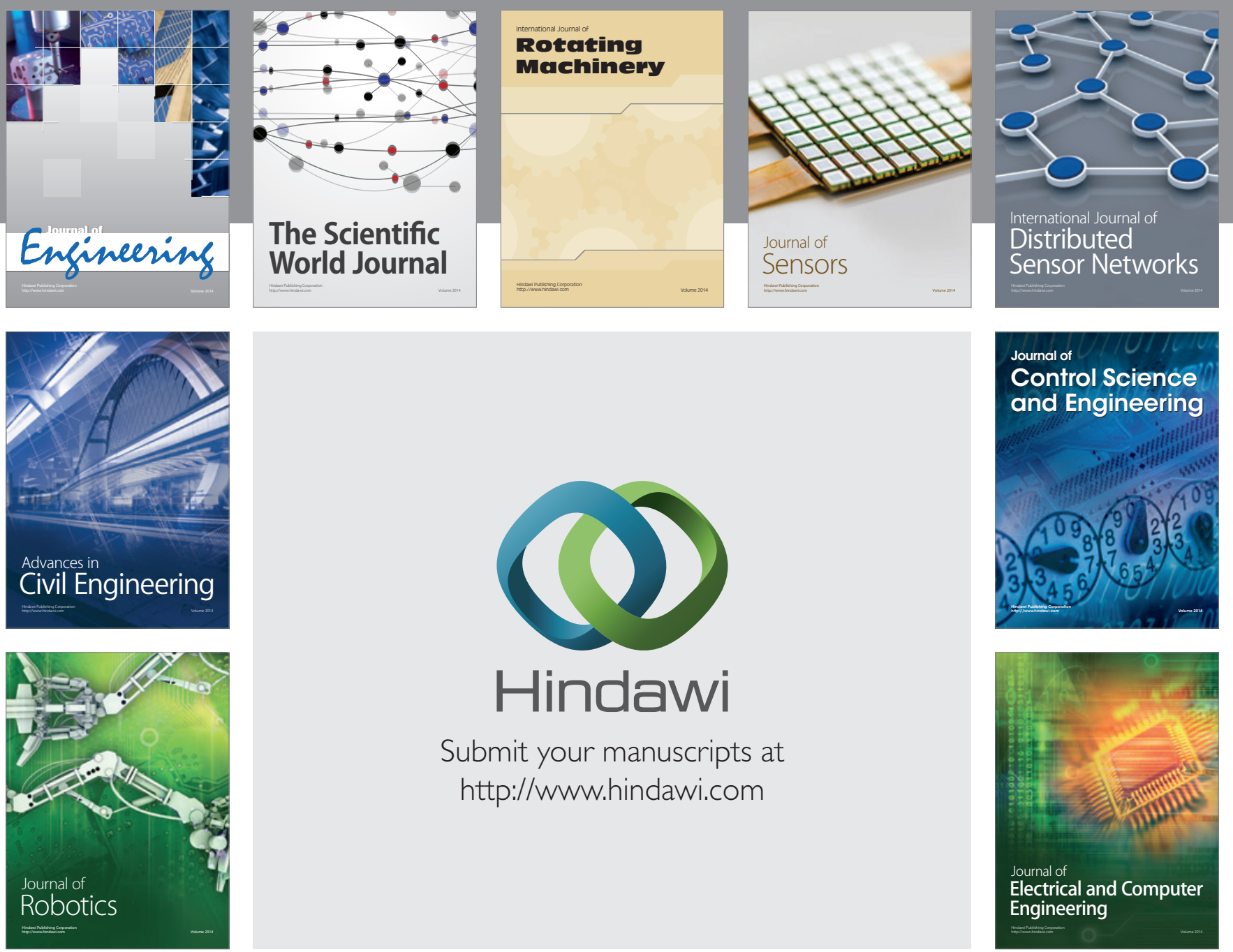

Submit your manuscripts at

http://www.hindawi.com
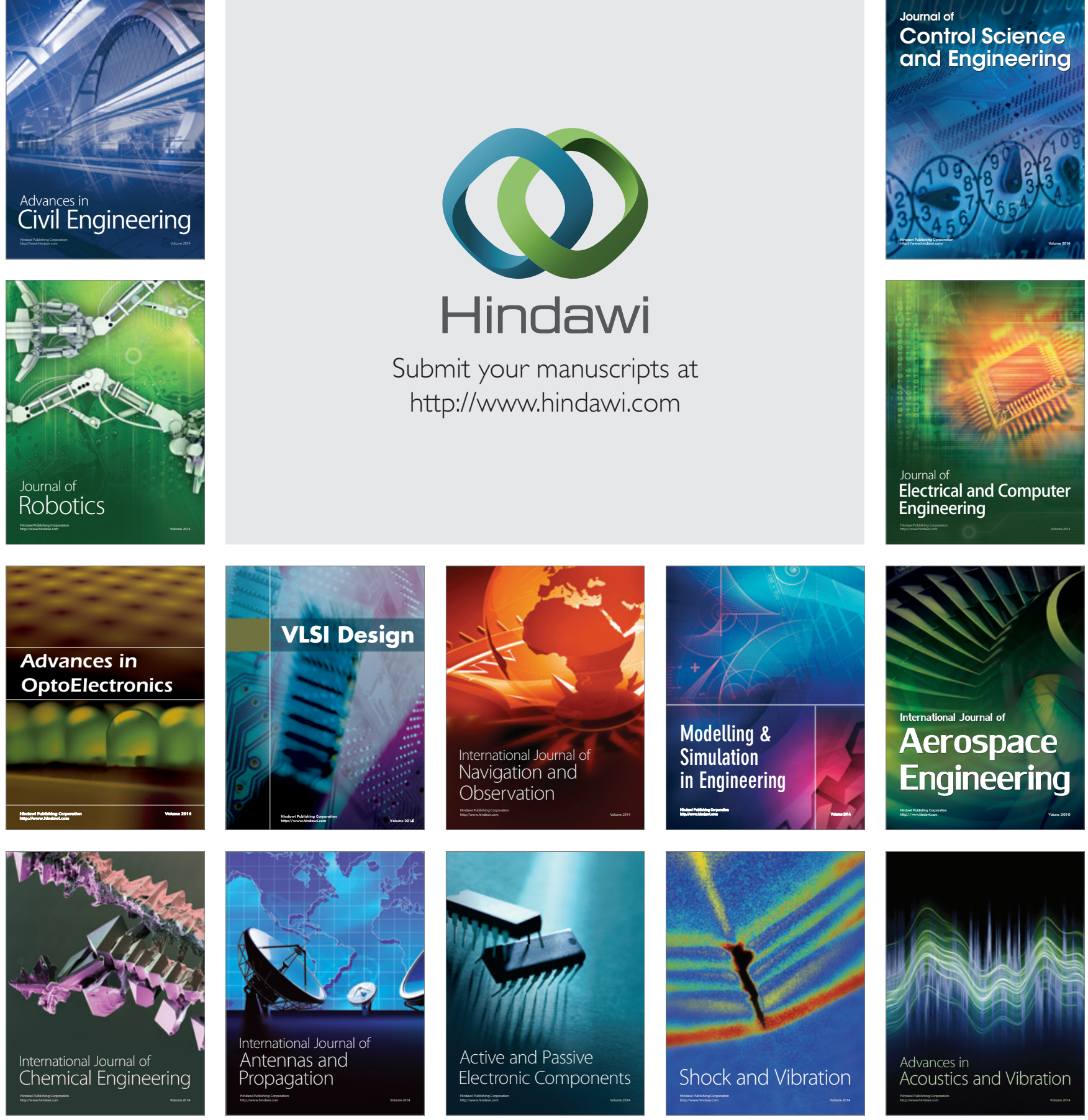